\title{
Youth mental health - service provision and planning for the future
}

\author{
L. Alexander ${ }^{1,2}$ and J. Lyne ${ }^{3,4, *}$ \\ ${ }^{1}$ Tallaght Adult Mental Health Services, Sheaf House, Tallaght, Dublin 24, Ireland \\ ${ }^{2}$ Department of Psychiatry, Trinity College Dublin, Dublin 2, Ireland \\ ${ }^{3}$ Royal College of Surgeons in Ireland, 123 St. Stephen's Green, Dublin 2, Ireland \\ ${ }^{4}$ North Dublin Mental Health Services, Ashlin Centre, Beaumont Road, Dublin 9, Ireland
}

Mental illness affects individuals throughout the lifespan, with first symptoms often emerging in early life. Mental health services for young people are faced with increasing demands, the impact of which on the delivery of all mental health services merits consideration. Much of the discourse in the current issue surrounds service planning in youth mental health services.

In their editorial, McMahon et al. (pp 163-167) discuss the future of youth mental health highlighting the necessity for integrated service planning, including the roles played by voluntary organisations and schools. The authors advocate for evidence-based education in schools for the prevention of mental health difficulties, which could supplement the existing curricula around well-being.

McMahon et al. also discuss transition between services as an important point of vulnerability for young people. They consider a paradigm shift in service transition where the cut-off age for service delivery is revised to accommodate societal need. This strategy has been adopted by Jigsaw, a youth mental health service providing support and brief intervention to clients between the ages of 12 and 25 years. Examining a further strand of transitional planning, Bond et al. (pp 187-193) describe the discharge care pathway for adolescents at transition age in an independent adolescent mental health service. Good engagement rates were noted among those referred onto an independent young adult mental health service. A high proportion of young people $(45.6 \%)$ were discharged back to primary care, with half of those discharged to primary care prescribed psychotropic medication at the point of discharge. This points to the need for training in primary care for prescribing to young people with mental illness.

In their retrospective chart review, Beatty et al. (pp 195-199) further reflect on the role of Jigsaw, as they report on referral pathways between Jigsaw and Galway Child and Adolescent/Adult Mental Health Services. Despite their distinct remits, there were few differences in the demographic or clinical profiles of patients referred from Jigsaw to Mental Health Services compared with those referred from Mental Health Services to Jigsaw. Echoing the point made by McMahon et al. about the importance of integrated service planning, Beatty et al. suggest that services may collaborate more effectively if staff are better educated about the remits and resources of the respective mental health services.

In their follow-up of the Child Cohort of the 'Growing up in Ireland' study, Cotter et al. (pp 177-185) compare psychopathology among groups of migrant and non-migrant cohorts. Other than moving house and country, migrant children were not found to experience more stressful life events, and there were few overall differences in the prevalence of psychopathology between the migrant and non-migrant groups. The authors provide an interesting discussion of the potential explanations for their findings, suggesting that better understanding of this apparent resilience among migrant youths may be able to inform public health strategies to prevent mental illness in young people.

This issue highlights that youth mental health is an area of interest for many stakeholders. Implementing service reform poses major challenges; however, it is important that we continue to evaluate how our services are delivered within the context of changing societal environments and needs. Taking a concerted approach to scrutinising and evaluating current practice, as undertaken by some of the authors herein, can help clinicians, researchers and policymakers to develop services with the goal of improving mental health for all across the lifespan.

\section{Conflict of interest}

LA and JL have no conflicts of interest to disclose.

*Address for correspondence: J. Lyne, North Dublin Mental Health Services, Ashlin Centre, Beaumont Road, Dublin 9, Ireland.

(Email: johnlyne@mail.com) 